\title{
The Disjunctive Decomposition of Logic Functions
}

\author{
Valeria Bertacco \\ Computer Systems Lab. \\ Stanford University
}

\author{
Maurizio Damiani \\ Synopsys, Inc. \\ Mountain View, CA
}

\begin{abstract}
We present an algorithm for extracting a disjunctive decomposition from the BDD representation of $F$. The output of the algorithm is a multiple-level netlist exposing the hierarchical decomposition structure of the function. The algorithm has thoretical quadratic complexity in the size of the input BDD. Experimentally, we were able to decompose most synthesis benchmarks in less than one second of CPU time, and to report on the decomposability of several complex ISCAS combinational benchmarks. We found the final netlist to be often close to the output of more complex dedicated optimization tools.
\end{abstract}

\section{Introduction}

The decomposition of a logic function $F\left(x_{1}, \cdots, x_{n}\right)$ is the identification of a set of functions $A_{i}\left(x_{i}\right)$ with no variables in common, and a function $L$ such that $[1,2,3]$ :

$$
F=L\left(A_{0}, \cdots, A_{i}, \cdots\right)
$$

The decomposition of a function is desirable for several reasons. First, it is obviously one method for passing from a flat representation of $F$ to a multiple-level one. It leads to a clustering of the inputs in simpler - and smaller - logic blocks, thereby reducing area and interconnect. This latter property is especially desirable in many implementation technologies, such as FPGAs or deep-submicron, where wiring resources are scarce or costly in terms of delay. Moreover, it exposes a parallelism in the computation of $F$, by evidencing the independence in the computation of the functions $A_{i}$. This parallelism can be exploited both in hardware, leading to faster realizations, as well as during simulation.

Because of its importance, decomposition is a classic subject of switching theory,still being researched $[1,2,3$, $4,5,6,7,8,9,10]$.

Known exact decomposition algorithms are based on the decomposition chart method of Ashenhust and Curtis [1]. The method is based on constructing trial partitions of the set of variables and then of verifying on the decomposition chart that $F$ can indeed be decomposed using that clustering of its inputs. Unfortunately, because of the potentially exponential number of trial partitions, exact decomposition is usually confined to functions of few variables. It is worth noting, however, that simplified techniques, such as algebraic factorization [4], have been extremely successful in transforming large two-level covers in multiple-level representations, and have been extended in various ways to include other forms of decomposition.
This paper presents two contributions. First, we present a novel, efficient exact decomposition procedure. The procedure is BDD-based, and it determines the decomposition of function directly from that of its cofactors. Its complexity is bounded by $|F|^{2} \times n_{F}$, where $|F|$ and $n_{F}$ denote the number of BDD nodes and input variables of $F$, respectively. In practice, we found the procedure to be very fast, as we were able to determine the decomposition of most benchmark circuits in less than five seconds on a PC. We have also derived -we believe for the first time- the decompositions of several combinational ISCAS benchmarks.

The decomposition engine is the core of a logic optimization tool, LODE. LODE outputs a netlist for a function $F\left(x_{1}, \cdots, x_{n}\right)$ based on its decomposition. If $F$ has no disjunctive decomposition, the output is constructed using the decomposition of the cofactors $F_{0}, F_{1}$ of $F$ with respect to its first variable, $x_{1}$. Interestingly, since the decomposition of $F$ is essentially unique [1], it could be shown that the netlist output by LODE is also canonical. Moreover, it is again generated in time proportional to $|F|^{2}$. Although LODE is currently missing many optimization opportunities, we found it in practice to produce netlists of comparable quality - sometimes better - to SIS, in a small fraction of the CPU time, for several synthesis benchmarks.

\section{Terminology.}

Hereafter, we assume the reader be familiar with BDDs $[11,12]$. Let $\mathcal{B}$ denote the Boolean set $\{0,1\}$. A logic function is a mapping $F: \mathcal{B}^{n} \rightarrow \mathcal{B}^{m}$. Hereafter, lower-case and upper-case letters will denote logic variables and functions, respectively. We will be mostly concerned with scalar functions $F: \mathcal{B}^{n} \rightarrow \mathcal{B}$. We use boldface to indicate vectorvalued functions. The $i^{\text {th }}$ component of a vector function $\mathbf{F}$ is indicated by $\mathbf{F}_{i}$.

We say that a function $F$ depends on a variable $x_{i}$ if $\partial F / \partial x_{i}[13]$ is not the constant function 0 . We call support of $F$ the set $S_{F}$ of variables $F$ depends on. The size of $S_{F}$ is the number of its elements, and it is indicated by $\left|S_{F}\right|$. Two functions $F, G$ are termed disjoint-support if they share no support variables, i.e. $S_{F} \cap S_{G}=\phi$.

\subsection{Disjunctive Decompositions.}

The decomposition of a function $F$ consists of finding other, simpler functions $L$ and $A_{i}$ such that

$$
F\left(x_{1}, \cdots, x_{n}\right)=L\left(A_{1}\left(x_{1}, \cdots\right), A_{2}\left(x_{1}, \cdots\right), \cdots\right)
$$

Definition 1. A function $L\left(a_{1}, \cdots, a_{k}\right), n>k \geq 2$ is said to reduce a function $F\left(x_{1}, \cdots, x_{n}\right)$ if there are $\bar{k}$ nonconstant disjoint-support functions $A_{1}, \cdots, A_{k}$ that satisfy 
Eq. (1). F is said to be prime if it cannot be decomposed by any $L$.

The functions $A_{i}$ will be termed formal inputs of the decomposition of $F$. The list of formal inputs to $F$ will be indicated as $F / L$, and termed decomposition list of $F$. We call disjunctive decomposition of $F$ any pair $(L, F / L)$ that satisfies Eq. (1).

If $F$ is decomposable, it is possible to characterize its decomposition as follows:

- there is a unique prime function $L$ decomposing it, up to permutations/complementations of its formal inputs.

- if $L$ has support size $\left|S_{L}\right|>2$, then also the functions in $F / L$ are uniquely determined, up to complementation/permutation.

- if $L$ has support size $\left|S_{L}\right|=2$ then $F$ is decomposable in exactly one of the following ways :

- as the OR of disjoint-support functions; the functions of $F / L$ are uniquely identified for the decomposition of finest granularity [14];

- as the complement of a OR-decomposed function ; again, the functions of $F / L$ are uniquely identified for the decomposition of finest granularity [14];

- as the XOR/XNOR of disjoint-support functions; the functions in $F / L$ are identified modulo complementation.

If a nontrivial function $A$ belongs to $F / L$, we take the cofactor $F_{A}$ (or $F(A=1)$ ) to be the function $L(A=$ $1, \cdots)$. Notice that, because of the uniqueness of $L$ and of the other elements in $F / L$, this function is unique and shares no variables with $A$.

\subsection{Representing decompositions.}

We represent the decomposition of the function $F$ rooted at a BDD node $N$ by annotating $N$ with a decomposition type field type; and a sorted list list of BDD nodes, representing the decomposition list of $F$. The field type can take on the values OR, NOR, XOR, XNOR, PRIME, UNDEF, depending on the type of decomposition. Initially, type is UNDEF. After application of the decomposition procedure, type can only take one of the other values.

Each node of list points to a BDD node. This node is the root of one of the formal inputs $A_{i}$.

The ambiguity due to permutations and complementations is resolved by sorting the functions according to their supports, and by not allowing complement edges in list.

Example 1. Consider the function $F=\operatorname{MAJORITY}(a \oplus$ $b, c+d, e f)$. Its BDD is shown in Fig.(1), along with the annotation of the root node and of significant other decompositions.

In order to resolve ambiguities in the representation, we adopt the following rules:

1. Lists are sorted by order of top variable, that is , a function $A_{i}$ appears before $A_{j}$ if the top variable of $A_{i}$ is ranked higher than that of $A_{j}$.

2. pointers to BDDs in decomposition lists of type XOR, XNOR, or PRIME cannot be complemented.

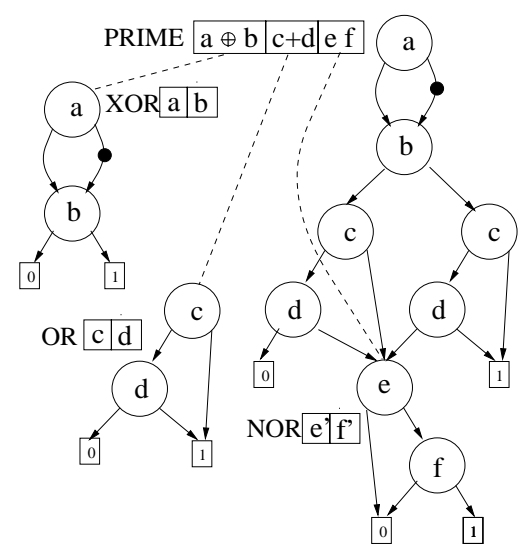

Figure 1. BDD and decomposition annotation for the function of Example (1).

As each function in the decomposition list $F / L$ may be itself decomposable, the lists associated with the decomposition of $F$ form a tree, hereafter called the decomposition tree of $F$.

\section{Shannon- and disjunctive- decompositions.}

A BDD node, with root variable $z$, represents a function $F\left(z, x_{1}, \cdots, x_{n}\right)$ as

$$
z^{\prime} F_{0}\left(x_{1}, \cdots, x_{n}\right)+z F_{1}\left(x_{1}, \cdots, x_{n}\right)
$$

where $F_{0}=F(z=0) ; \quad F_{1}=F(z=1)$. Our goal is to infer a decomposition

$$
F=L(A, B, \cdots)
$$

from the decompositions of $F_{0}, F_{1}$. This inference mechanism is the basis of the decomposition algorithm of Section (4).

The link between the decomposition of $F$ and that of its cofactors is conceptually simple. For example, if a function $A$ belongs to the decomposition tree of both $F_{0}$ and $F_{1}$, then it must belong to the decomposition tree also of $F$. Unfortunately, several cases and sub-cases need be considered in practice. These are enumerated below.

We now describe in detail how the decomposition structure of $F$ is reflected in that of its cofactors. Moreover, we show how the information on the decomposition of the two cofactors is sufficient to infer the decomposition of $F$.

The top variable $z$ of $F$ can appear in the decomposition of a function $F$ in only one of the four following ways :

$1: F$ is the OR/NOR of $z / z^{\prime}$ with a function $A, z \notin S_{A}$;

$2: F=z \oplus A, z \notin S_{A}$.

3 : Cases 1 and 2 do not hold, and $z$ belongs to the support of one formal input of $F$ (say, $A$ ), with $\left|S_{A}\right| \geq 2$;

4 : Cases 1 and 2 do not hold, and $z$ is one of the formal inputs of $F$.

We now analyze the consequences of these cases on the decomposition of $F_{0}, F_{1}$.

Case 1.

In this case, one of $F(z=0), F(z=1)$ is a constant. Viceversa, suppose that one cofactor is a constant (say, 
$F_{1}=1$, the other cases being symmetric). In this case, trivially $F=F_{0}+z$, hence $\mathrm{F}$. type $=\mathrm{OR}$ and the decomposition list of $F$ is that of $F_{0}$ plus $z$.

\section{Case 2.}

Clearly, in this case, $F_{0}=F_{1}^{\prime}$. Verifying $F_{0}=F_{1}^{\prime}$ is sufficient to infer the decomposition of $F$ as $F=z \oplus F_{0}$.

\section{Case 3.}

Suppose $z$ belongs to the support of a function (say, $A$ ) with nontrivial support $\left|S_{A}\right| \geq 2$. We need to distinguish two sub-cases :

3.a Neither cofactor of $A\left(A_{z=0}, A_{z=1}\right)$ is a constant;

3.b Exactly one cofactor of $A$ is a constant.

Notice that the case of both cofactors being constants rules out the possibility that $\left|S_{A}\right| \geq 2$.

\section{Case 3.a}

In this case, $F_{0}, F_{1}$ are :

$F_{0}=L(A(z=0), B, \cdots) ; F_{1}=L(A(z=1), B, \cdots)$

In other words, $F_{0}$ and $F_{1}$ are decomposable by the same function $L$. Moreover, the decomposition lists of $F_{0}$ and $F_{1}$ will coincide, except for at most one element $(A(z=0)$ vs. $A(z=1))$.

Viceversa, suppose that $F_{0}$ and $F_{1}$ have decompositions containing functions $A_{0}$ and $A_{1}$, respectively, and such that

$$
\begin{aligned}
& F_{0}\left(A_{0}=0\right)=F_{1}\left(A_{1}=0\right) \text { and } \\
& F_{0}\left(A_{0}=1\right)=F_{1}\left(A_{1}=1\right)
\end{aligned}
$$

it then follows that, by forming $A=z^{\prime} A_{0}+z A_{1}$, one can write

$$
F=F_{0}(A, \cdots)=F_{1}(A, \cdots)
$$

Example 2. Consider function $F=\operatorname{MAORITY}(\bar{a} b+$ $a d, c+e, f g)$, and its cofactors $F_{0}=F(a=0), F_{1}=$ $F(a=1)$. Both cofactors are annotated as PRIME functions, with decomposition lists $b, c+e, f g$ and $c+e, d, f g$, respectively. The two lists differ in exactly one element ( $b$ instead of $d$ ), and moreover, Eq. (5) holds for $A_{0}=$ $b, A_{1}=d$. Hence the inference of the decomposition $\bar{a} b+a d, c+e, f g$ for $F$.

Example 3. Consider the function $F=M A J O R I T Y(a \oplus$ $b, c+e, f g)$. In this case, $F_{0}$ and $F_{1}$ have identical decomposition lists $b, c+e, f g$. We infer the decomposition by observing that the only possibility is that in Eq. (5), $A_{1}=A_{0}^{\prime}$. We then test Eq. (5) using first $A_{0}=b, A_{1}=b^{\prime}$. Since the test succeeds, we list $A=z^{\prime} A_{0}+z A_{1}=z \oplus A_{0}$ in the decomposition list of $F$, along with $c+e, f g$.

\section{Case 3.b}

We only consider the case where the cofactor $A(z=1)=1$ (i.e. $A=z+A_{0}$ ), the other cases being symmetric. Then

Suppose that $F$ has decomposition $F=L(A, B, C, \cdots)$.

$$
F_{0}=L\left(A_{0}, B, C, \cdots\right) ; \quad F_{1}=L(1, B, C, \cdots) .
$$

In particular, the element $A_{0}$ is missing from the decomposition of $F_{1}$, and $F_{0}\left(A_{0}=1\right)=F_{1}$.
Viceversa, suppose that $F_{0}$ has a decomposition containing a function $A_{0}$, that $S_{A_{0}} \cap S_{F_{1}}=\phi$, and that $F_{0}\left(A_{0}=1\right)=F_{1}$. In this case, one can write

$$
F=F_{0}\left(A_{0}+z, \cdots\right) \text {. }
$$

Example 4. Consider the function $F=M A J O R I T Y(a+$ $b, c, d+e)$. Consider the cofactors $F_{0}=F(a=0)=$ $M A J O R I T Y(b, c, d+e)$ and $F_{1}=F(a=1)=c+d+e$. In this case, $b$ is the only function of $F_{0}$ (just a variable, in this case) not in the support of $F_{1}$. The test $F_{0}(b=1)=F_{1}$ is satisfied; hence $A=a+b$ and the decomposition list of $F$ is that of $F_{0}$ with $A$ replacing $b$.

\section{Case 4.}

$F$ must be of type PRIME, and the function $z$ belongs to its list of formal inputs. The decomposition tree - and hence the decomposition list - of $F$ can be constructed from those of $F_{0}, F_{1}$ as follows:

1. If a function $A$ belongs to the decomposition tree of $F_{0}$ and $S_{A} \cap S_{F_{1}}=\phi$, then $A$ belongs to the decomposition tree of $F$, along with its descendants.

2. If a PRIME function $A$ belongs to the decomposition tree of $F_{0}, F_{1}$, then it belongs to that of $F$, along with all its descendants.

3. If a OR/NOR function $A$ belongs to the decomposition tree of $F_{0}$, and a OR/NOR function $B$ belongs to the decomposition tree of $G$, then the OR of the functions common to the decomposition lists of $A$ and $B$ belongs to the tree of $F$;

4. If a XOR function $A$ is in the tree of $F_{0}$, and a XOR function is in the tree of $F_{1}$, then the XOR of the common terms belongs to the tree of $F$.

5. The decomposition list of $F$ contains all functions obtained by rules 1-4 that do not have ancestors.

Rule (3) above accounts for the fact that if a function $A=$ $a+b+c$ decomposes $F_{0}$ and, say $B=a+b+d$ decomposes $F_{1}$, then $a+b$ certainly decomposes $F$. Similarly for rule (4).

Example 5. Consider the function $F=a^{\prime}(b+c)(d+e+$ $f)+a(b+c+d)(e+f+g)$. By rule (1), the function $g$ belongs to the tree of $F$. By rule (3), the functions $b+c$ and $e+f$ belong to the decomposition of $F$. Eventually, the function $d$ belongs to the decomposition of $F$ by Rule (2). The decomposition list of $F$ is then $a, b+c, d, e+f, g$.

Rules 1-5 are sufficient to construct the decomposition tree/list of $F$ :

Theorem 1. If a function A belongs to the decomposition tree of $F$, then one of conditions $1-4$ above must hold true for $A$.

\section{A BDD-based decomposition procedure.}

In this section we present the algorithm for annotating the decomposition of a function $F$ on its BDD representation. The annotation is accomplished in a single sweep of 


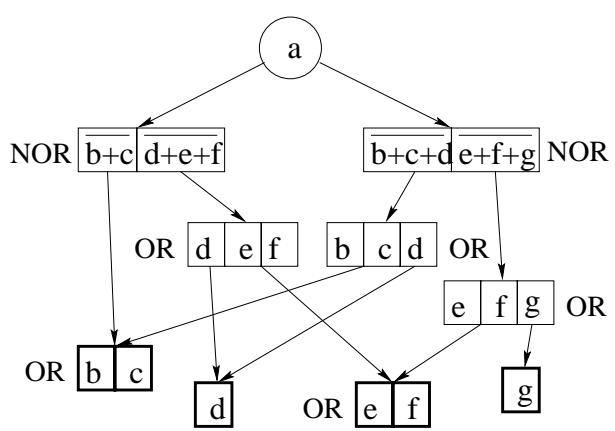

Figure 2. Construction of the decomposition tree for the Example (5).

the BDD nodes, sorted topologically in a linear array. During the sweep, each node is inspected, and the decomposition of the function rooted at that node is determined, from that of the children. The node is then labeled with a pointer to its decomposition list and annotated with its decomposition type.

The decomposition method is enumerative. We try to verify Cases 1 to $3 b$ of Section (3), in order. If we fail, a node is annotated PRIME and its decomposition list constructed as in Case 4.

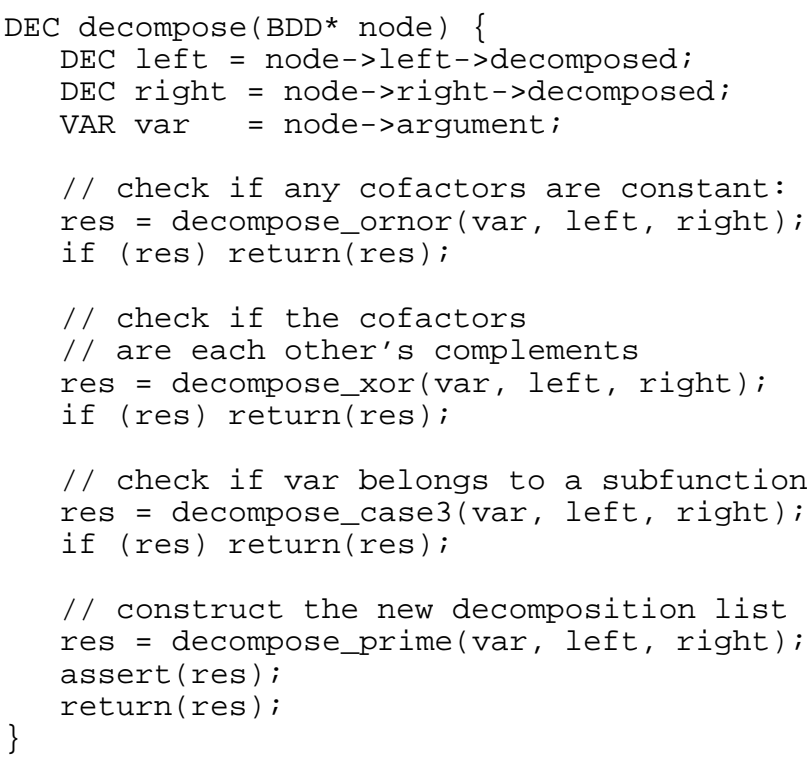

\subsection{Synthesis.}

LODE generates a netlist from a depth-first traversal of the decomposition of $F$ as follows:

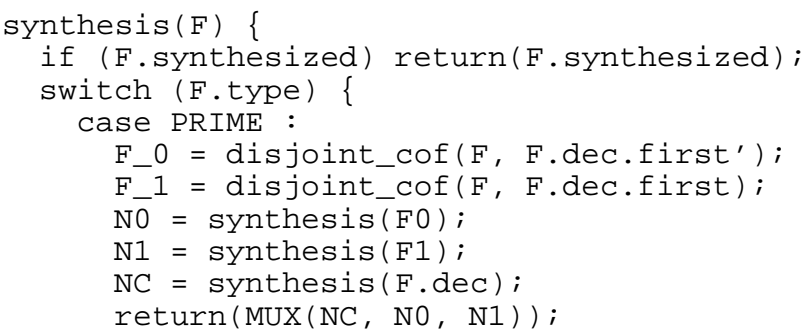

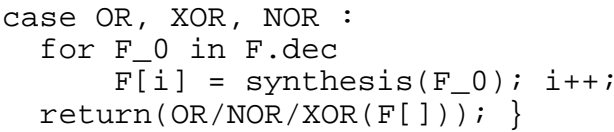

\section{Experimental results.}

The procedures of this paper have been implemented in $\mathrm{C}$ and tested on several combinational logic benchmarks. The CPU time was taken on a PC equipped with a $150 \mathrm{MHz}$ Pentium and 96Mbyte of main memory.

Table (1) below reports results on the decomposability of some large combinational and sequential benchmarks. Column DEC reports the number of decomposable outputs, while CPU time is reported in seconds. The decompositions themselves are often of interest. For instance, in some sequential circuits, such as s1494, the next-state outputs are often ANDed with the same signal (v13_D_0C). Most of the outputs of C880 contain complex functions of the same formal input AND(72GAT[12], 68GAT[11], 13GAT[2], 73GAT[13]). The output is also often the OR of these complex functions with 255GAT[54], 259GAT[55], etc... Synthesis benchmarks are mostly decomposable. The decomposition of some circuits, such as DES, pair, etc ... also evidences regular designs, that we cannot describe here for reasons of space. For some ISCAS circuits, we have also been able to decompose some of the outputs corresponding to complex functions.

Table (2) below compares the literal counts obtained by LODE against those obtained by SIS running rugged_script. Finally, table (3) reports the CPU time employed by LODE for the decomposition.

For the largest benchmarks, the limited set of BDD transformations of LODE do not compensate for the exceptional growth of the BDD representation with respect to the original representation. We are currently working towards a partitioning strategy and enriching the transformation set.

\section{Conclusions.}

We have presented an algorithm for the disjunctive decomposition of logic functions, starting from a BDD representation. The algorithm has worst-case complexity quadratic in the BDD size. We found it very fast in practice, as we were able to obtain the decomposition of all benchmark functions in a few minutes in the worst case. We have been able to present results on the decomposability of complex benchmarks for the first time. Moreover, we have been able to generate a very compact, canonical multiple-level circuit directly from a BDD representation.

We are currently investigating some implications of the present work: First, the possibility of generating the decompositio form of this paper directly from a netlist. Second, we are exploring the applicability of this representation to rapid prototyping, technology mapping and reachability analysis.

\section{References}

[1] R. Ashenhurst. The decomposition of switching functions. In Proceedings of the International Symposium on the Theory of Switching, pages 74-116, April 1957.

[2] J. P. Roth and R. M. Karp. Minimization over boolean graphs. IBM Journal, pages 661-664, April 1962. 


\begin{tabular}{|l|r|r|r|r|}
\hline Circuit & Inputs & Outputs & DEC & CPU time \\
\hline C1355 & 41 & 32 & 0 & $91.25 \mathrm{~s}$ \\
C1908 & 33 & 25 & 7 & $7.58 \mathrm{~s}$ \\
C3540 & 50 & 22 & 0 & $21.10 \mathrm{~s}$ \\
C432 & 36 & 7 & 1 & $1.23 \mathrm{~s}$ \\
C499 & 41 & 32 & 0 & $83.47 \mathrm{~s}$ \\
C880 & 60 & 26 & 25 & $2.71 \mathrm{~s}$ \\
CM42 & 4 & 10 & 10 & $0.15 \mathrm{~s}$ \\
CM85 & 11 & 3 & 3 & $0.27 \mathrm{~s}$ \\
alu4 & 14 & 8 & 4 & $0.37 \mathrm{~s}$ \\
apex6 & 135 & 99 & 99 & $2.62 \mathrm{~s}$ \\
apex7 & 49 & 37 & 36 & $1.03 \mathrm{~s}$ \\
comp & 32 & 3 & 3 & $0.71 \mathrm{~s}$ \\
count & 35 & 16 & 16 & $0.73 \mathrm{~s}$ \\
frg2 & 143 & 139 & 126 & $2.86 \mathrm{~s}$ \\
k2 & 45 & 45 & 35 & $1.04 \mathrm{~s}$ \\
pair & 173 & 137 & 125 & $4.02 \mathrm{~s}$ \\
rot & 135 & 107 & 77 & $22.62 \mathrm{~s}$ \\
vda & 17 & 39 & 20 & $0.40 \mathrm{~s}$ \\
x3 & 135 & 99 & 99 & $2.69 \mathrm{~s}$ \\
x4 & 94 & 71 & 65 & $1.90 \mathrm{~s}$ \\
apex1 & 45 & 45 & 35 & $1.01 \mathrm{~s}$ \\
apex2 & 38 & 3 & 3 & $1.14 \mathrm{~s}$ \\
apex4 & 9 & 19 & 4 & $0.33 \mathrm{~s}$ \\
apex5 & 114 & 88 & 82 & $2.34 \mathrm{~s}$ \\
e64 & 65 & 65 & 64 & $1.31 \mathrm{~s}$ \\
misex2 & 25 & 18 & 17 & $0.57 \mathrm{~s}$ \\
seq & 41 & 35 & 34 & $1.10 \mathrm{~s}$ \\
s1196 & 32 & 32 & 14 & $0.71 \mathrm{~s}$ \\
s1238 & 32 & 32 & 14 & $0.75 \mathrm{~s}$ \\
s1423 & 91 & 79 & 71 & $12.48 \mathrm{~s}$ \\
s1488 & 14 & 25 & 19 & $0.36 \mathrm{~s}$ \\
s1494 & 14 & 25 & 19 & $0.34 \mathrm{~s}$ \\
s420 & 35 & 18 & 18 & $0.75 \mathrm{~s}$ \\
s444 & 24 & 27 & 21 & $0.54 \mathrm{~s}$ \\
s641 & 54 & 42 & 35 & $1.12 \mathrm{~s}$ \\
s953 & 45 & 52 & 22 & $20.97 \mathrm{~s}$ \\
\hline
\end{tabular}

Table 1. Decomposability of some large benchmark circuits.

\begin{tabular}{|l|r|r||r|r|r|}
\hline Circuit & IN & OUT & $\begin{array}{r}\text { Original } \\
\text { literals }\end{array}$ & $\begin{array}{r}\text { LODE } \\
\text { literals }\end{array}$ & $\begin{array}{r}\text { SIS } \\
\text { literals }\end{array}$ \\
\hline 9symml & 9 & 1 & 277 & 76 & 223 \\
CM150 & 21 & 1 & 77 & 47 & 51 \\
PARITY & 16 & 1 & 60 & 60 & 60 \\
alu2 & 10 & 6 & 453 & 354 & 357 \\
cmb & 16 & 4 & 62 & 36 & 51 \\
f51m & 8 & 8 & 169 & 98 & 91 \\
lal & 26 & 19 & 221 & 134 & 105 \\
mux & 21 & 1 & 92 & 47 & 51 \\
term1 & 34 & 10 & 624 & 165 & 197 \\
ttt2 & 24 & 21 & 341 & 258 & 216 \\
s1494 & 14 & 25 & 1393 & 793 & 661 \\
s298 & 17 & 20 & 244 & 146 & 114 \\
s526 & 24 & 27 & 445 & 257 & 191 \\
s832 & 23 & 24 & 769 & 431 & 352 \\
\hline
\end{tabular}

Table 2. Experimental result and comparisons against SIS.

\begin{tabular}{|l|r|r||r|r|}
\hline Circuit & IN & OUT & $\begin{array}{r}\text { LODE } \\
\text { CPU time }\end{array}$ & $\begin{array}{r}\text { SIS } \\
\text { CPU time }\end{array}$ \\
\hline 9symml & 9 & 1 & $0.26 \mathrm{~s}$ & $25.83 \mathrm{~s}$ \\
CM150 & 21 & 1 & $0.51 \mathrm{~s}$ & 0.51 \\
PARITY & 16 & 1 & $0.38 \mathrm{~s}$ & 0.27 \\
alu2 & 10 & 6 & $0.28 \mathrm{~s}$ & $106.56 \mathrm{~s}$ \\
cmb & 16 & 4 & $0.36 \mathrm{~s}$ & 0.31 \\
f51m & 8 & 8 & $0.26 \mathrm{~s}$ & 2.23 \\
lal & 26 & 19 & $0.55 \mathrm{~s}$ & 2.21 \\
mux & 21 & 1 & $0.48 \mathrm{~s}$ & 0.55 \\
term1 & 34 & 10 & $0.75 \mathrm{~s}$ & $16.22 \mathrm{~s}$ \\
ttt2 & 24 & 21 & $0.55 \mathrm{~s}$ & 5.10 \\
s1494 & 14 & 25 & $0.34 \mathrm{~s}$ & $65.77 \mathrm{~s}$ \\
s298 & 17 & 20 & $0.40 \mathrm{~s}$ & 1.83 \\
s526 & 24 & 27 & $0.52 \mathrm{~s}$ & 5.62 \\
s832 & 23 & 24 & $0.54 \mathrm{~s}$ & $27.66 \mathrm{~s}$ \\
\hline
\end{tabular}

Table 3. CPU time for decomposition and comparisons against SIS.

[3] H. A. Curtis. A New Approach to the Design of Switching Circuits. Van Nostrand, Princeton, N.J., 1962.

[4] R.K. Brayton and C. McMullen. The decomposition and factorization of boolean expressions. In ISCAS, Proceedings of the International Symposyium on Circuits and Systems, pages 49-54, 1982.

[5] R. Murgai, Y. Nishizaki, N. Shenoy, R.K. Brayton, and A. Sangiovanni-Vincentelli. Logic synthesis fro programmable gate arrays. In Proceedings 27th ACM/IEEE Design Automation Conference, pages 620-625, June 1990.

[6] Kevin Karplus. Representing boolean functions with if-thenelse dags. Technical Report UCSC-CRL-88-28, Baskin Center for ComputerEngineering \& Information Sciences, 1988.

[7] Kevin Karplus. Using if-then-else dsgs for multi-level logic minimization. Technical Report UCSC-CRL-88-29, Baskin Center for ComputerEngineering \& Information Sciences, 1988.

[8] Kevin Karplus. Using if-then-else dsgs to do technology mapping for field-programmable gate arrays. Technical Report UCSC-CRL-90-43, Baskin Center for ComputerEngineering \& Information Sciences, 1990.

[9] K.-R. Pan Y.-T.Lai and M. Pedram. Obdd-based functional decomposition: algorithms and implementation. IEEE Trans. on CAD/ICAS, 15(8):977-990, August 1996.

[10] T. Sasao. Multiple-valued decomposition of generalized boolean functions and the complexity of programmable logic arrays. IEEE Trans. on Computers, C-30(9):635-643, September 1981.

[11] R. E. Bryant. Graph-based algorithms for boolean function manipulation. IEEE Trans. on Computers, 35(8):677-691, August 1986.

[12] K. S. Brace, R. L. Rudell, and R. E. Bryant. Efficient implementation of a BDD package. In Proc. DAC, pages 40-45, June 1990.

[13] Edward J. McCluskey. Logic Design Principles With Emphasis on Testable Semicustom Circuits. Prentice-Hall, 1986.

[14] V. Bertacco and M. Damiani. Boolean function representation based on disjoint-support decompositions. submitted to ICCD 96, to appear, October 1996.

[15] O. Coudert and J.C. Madre. A unified framework for the formal verification of sequential circuits. In Proc. ICCAD, pages 126-129, November 1990. 